\title{
Cognitive Linguistics-Inspired Empirical Study of Chinese EFL Teaching
}

\author{
Youmei Gao \\ Department of Translation, School of English Studies, Tianjin Foreign Studies University, Tianjin, China. \\ Email: gaoyoumei@tjfsu.edu.cn \\ Received July 23 ${ }^{\text {rd }}$, 2011; revised August 28 $8^{\text {th }}$, 2011; accepted September $4^{\text {th }}, 2011$.
}

\begin{abstract}
Aiming to verify how and why insightfulness of the CL-inspired approach can facilitate L2 or FL learners in the process of learning English as a second or a foreign language, an empirical study of Chinese EFL learners has been carried out in the authentic Chinese EFL classroom on the campus of Tianjin Foreign Studies University. After the classroom treatment, the EG outperformed the CG by $21.7 \%$ based on the results from the delayed posttest in Experiment 1; by 6.73\% in Experiment 2; by 6.61\% in Experiment 3; by 21.7\% in Experiment 4. The study has concluded that through awakening students' metaphoric awareness and analyzing features of the image schematic structure, the CL-inspired approach benefits L2 or FL learners in the process of learning English as a foreign language with respect to lexical and grammatical competence, metaphorical and pragmatic or sociolinguist competence, including semantic comprehension in reading, writing and translation, particularly in terms of long-term retention of memory.
\end{abstract}

Keywords: Cognitive Linguistic Approach, Chinese EFL Teaching

\section{Introduction}

The cognitive framework has been recently reported to be able to help L2 or FL learners acquire the target materials in an effective manner and achieve long-term retention in memory. Experimental evidence (e.g. Kövecses \& Szabó, 1996; Boers, 2000a, 2000b) and theoretical contributions (e.g. Boers, 1999, Boers \& Demecheleer, 2004; Lazar, 1996; Littlemore, 2001a) suggest that the cognitive framework can indeed assist L2 or FL learners to acquire figurative expressions in an effective manner and achieve long-term retention in memory. Nevertheless, how it works in the authentic Chinese university EFL classrooms remains as a not or at least not fully touched upon area until very recently.

It has been noted that on the first hand linguists with the background of generative linguistics proposed "learnability" instead of "teachability", on the other hand, the problematic issue of how to present the way of linguistic input for the L2 or FL learner to become a successful learner has not been resolved yet (Liu, 2010). The more puzzling situation is that despite the fact that the significance of lexical learning is fully agreed in the foreign language world, yet the desirable proposal for how the lexis can be better acquired, particularly in terms of advanced usage such as metaphors, set phrases, idioms and proverbs is still not available (Gao, 2010).

\section{Literature Review}

Putting Cognitive Linguistics and applied linguistics together, in a narrow sense, referring to applying Cognitive Linguistics theory to L2 or FL acquisition, is a newly-emerged research area; nevertheless, scholars domestically and internationally have achieved significant progress in this field. Sophia Skoufaki (2008) states, in his "Conceptual metaphoric meaning clues in two idiom presentation methods” (pp. 133-158), that metaphoric studies of idioms in L2 or FL acquisition started with an empirical study by Kövecses, Zoltán \& Szabó (1996), which focuses on whether memory of lexes can be enhanced with explanation of conceptual metaphor theory (CMT). Lynne Cameron \& Graham Low (1999) propose a framework for metaphor studies in their book Researching and Applying $\mathrm{Me}$ - taphor, thus introducing metaphor studies into applied linguistic research area. Frank Boers \& Murielle Demecheleer (1998) put forward, in their "A cognitive semantic approach to teaching prepositions”, which examines possible ways in which cognitive semantic analyses of prepositions could be used to anticipate comprehension problems, and facilitate comprehension of unfamiliar figurative senses. According to them, the figurative senses of a preposition are extended from its spatial senses through conceptual metaphors, thus in a pedagogical context it may be useful to draw learners' attention to those aspects of a preposition's spatial sense that are especially relevant for its metaphorization processes (pp. 197-204). Boers F. (2000) conducted an empirical experiment and concludes that the metaphorical themes or source domains facilitate retention of unfamiliar figurative expressions, thus, he proposes that classroom activities should be focused on enhancing FL learners' metaphorical awareness for facilitating their vocabulary acquisition (pp. 553-571). Boers \& Lindstromberg (2000) edited a book entitled Cognitive Linguistic Approaches to Teaching Vocabulary and Phraseology, which includes a number of empirical studies of application of the CL theories to L2 instruction, such as Beréndi, Márta (pp. 65-100) Csábi, Szilvia and Kövecses, Zoltán's (pp. 65-100) empirical study of CMs and metonymies in vocabulary teaching; Condon, Nora's (pp. 133158) study of CL pedagogy in learning phrasal verbs, etc. Littlemore (2001).

Moreover, Jeannette Littlemore (2001) explores L2 acquisition in her empirical study of the cognitive style and the use of communication strategy, which provides suggestive implication for the research in this area. In her "Metaphoric Competence, Second Language Learning, and Communicative Language Ability”, Littlemore \& Low (2006) argues that the metaphoric competence can contribute centrally to grammatical, textual, illocutionary, sociolinguistic, and strategic competence. She 
also maintains that metaphor is highly relevant to L2 learning, teaching and testing, from the earliest to the most advanced stages of learning.

Additionally, there is no denying that Frank Boers has made important contributions to this field. Boers (1998: pp. 197-204), advocates that conceptual metaphor theory (CMT) is efficient in teaching English prepositions. According to him, how a cognitive semantic analysis of a preposition can be used to anticipate comprehension problems about an area of perennial difficulty for learners and to facilitate greater comprehension of unfamiliar uses.

When it comes to CL application in the field of applied linguistics (AL), the following achievements have been made in China. Gao (2010) in her book: Applying Cognitive Linguistics to $L 2$ Acquisition speculates on the effectiveness of cognitive linguistics-inspired methodology in the process of L2 or FL acquisition. In her paper "Comparative Research on Preferences of Learning Style”, she (2001) argues that "individuals all have basic capacity to learn and to teach, nevertheless, they are not all able to learn and teach effectively in the same exactly way", she (1996) discusses the cognitive context and its significance in text reading. Gao (2011) notes that multidimensional approach to the "non-arbitrary nature" of linguistic sign provides the desirable answer to the nature of language, which can not be answered from the view of "arbitrariness" of language, essentially challenging the "autonomy" theory of syntax structure and bears great potential to L2 or FL teaching and research; $\mathrm{Li}$ (2004) has conducted a comprehensive study of applying the CMs (conceptual metaphors) and the IMS (image schemas) in English learning. In his book Applied Cognitive Linguistics, Li provides an intensive discussion on the effect of the CMs and IMs on EFL teaching and learning, which is very encouraging; Liang (2000) has explored Enlightenments of the Cognitive Linguistics on English Vocabulary Teaching, which is very suggestive; Wang, Yin \& Li, Hong (2004) in A Survey of Cognitive Linguistics speculate on the application of metaphor to FL teaching at three levels and put forward the view of "combining three into one", which refers to competence of language, communication, and metaphoric thinking; In his "Cognitive linguistic view of language and principles for FL teaching”, Liu (2010) points out differences and inadequacies in pedagogy resulting from the view of language acquisition, since principle of language teaching lies in the attitude towards the process of language acquisition.
Briefly, scholars in the West and in China have achieved significant results in the field of the foreign language teaching and research, shifting their traditional views of language competence to cognitive approaches to the motivation of language. Nevertheless, how these strategies work in the authentic Chinese university EFL classrooms in terms of advanced usage of English lexis remains as a not fully touched upon until very recently. Motivated under the situation mentioned above, an empirical study of Chinese EFL learners has been carried out in the authentic Chinese EFL classroom on the campus of Tianjin Foreign Studies University, with an aim to verify the validness of the proposed cognitive-inspired approach to L2 of FL acquisition, thus bridging the gap between the cognitive linguistic researches and applied linguistic studies.

\section{Rational of Present Research}

The present study contends that the major advantage of the CL methodology is that it reveals the hidden relationships that run among many metaphorical and metonymic expressions, idioms and proverbs, and verb-particle constructions as well. The evidence experimentally tested throws light on the systematicity and the re-occurring conceptual metaphors and image schemas that have an impact on the way we act, think and express our inner thoughts. That's why it can help L2 or FL learners categorize metaphorical sense of words or linguistic expressions under certain conceptual domains and organize linguistic realizations of CMs and IMs according to the cognitive mechanisms. This study also contends that awareness that EFL need not be learned entirely via a long and daunting road of blind memorization must be an encouraging thought to learners who recognize that large segments of the target language actually make sense. This encouragement can lead to positive affect, which is obviously beneficial to longer-term learning behavior (Arnold, 1999; also refer to the survey by the current research in the assessment of the approaches used for this present study). The CL-inspired approach adopted in the present study has been proposed based on the insightfulness of the CL theories, i.e. conceptual metaphor theory (CMT) and the image schema theory (IMT), which is supported with the dual coding theory (DCT) and the trace theory of memory, The fundamental basis for the proposed CL-inspired approach (Figure 1) can be presented in the following framework.

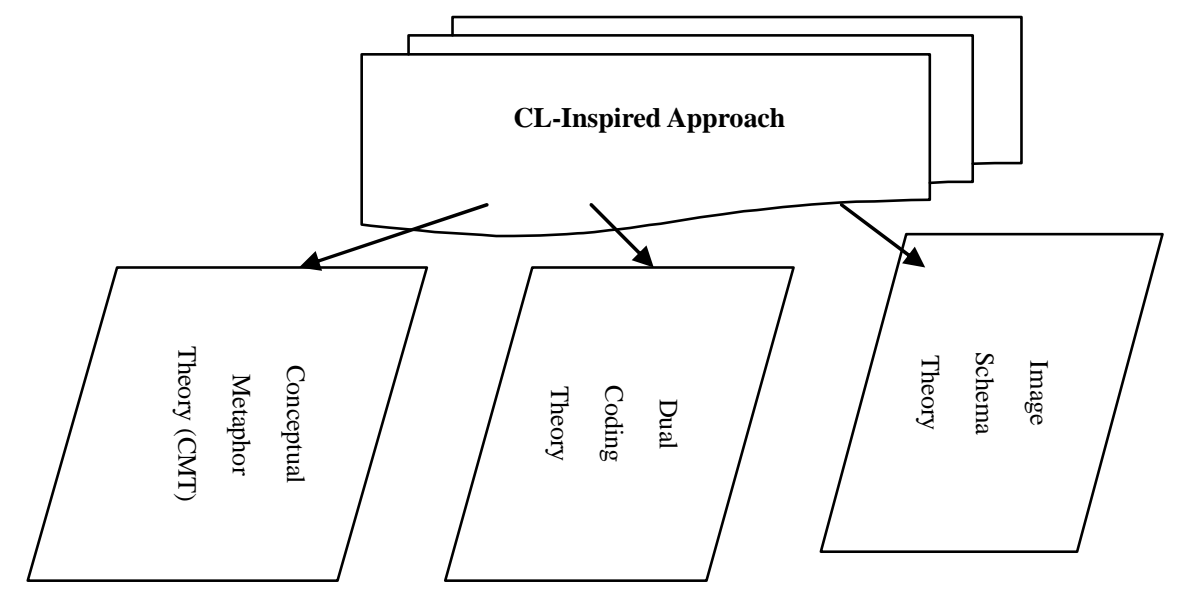

Figure 1.

The fundamental basis for CL-inspired approach. 


\section{Methodology: An Empirical Study of Chinese EFL Learners}

\section{The Objective}

This study essentially makes an attempt to verify what contribution cognitive linguistics (CL) could make to the development of applied linguistics (AL), thus bridging the gap between the CL theory and its application to the field of the AL, focusing on the primary research question, that is, in which way and to what extent the CL-inspired approach benefits EFL learners in the process of learning English as a L2 or a FL. The major hypothesis is that the CL-inspired pedagogy facilitates $L 2$ or FL acquisition in terms of advanced usage of vocabulary, including metaphorical sense of words, verb-particle structures, idioms and proverbs with respect to comprehension and retention of memory, thus enhancing metaphorical competence, pragmatic or socio-linguistic competence, and positive affect.

To verify effectiveness of the CL-inspired approach on the L2 or FL acquisition and to measure the long-term, mid-term and short-term recall of the target materials, a series of the four classroom experiments followed by a series of delayed and immediate posttests and pretests will be conducted, which is designed and implemented by the present researcher.

\section{Methods and Approaches}

To verify the primary hypothesis related to the question mentioned, a series of four classroom experiments were carried out with four categories of target materials: lexical metaphors for Experiment 1; verb-particle constructions for Experiment 2; idioms for Experiment 3; proverbs for Experiment 4.

During the whole process of the classroom experiments, four handouts were presented: Handout 1 contained 20 items of lexical metaphors for Experiment 1; Handout 2, 20 items of verbal-particle (VP) constructions for Experiment 2; Handout 3, 20 items of idioms for Experiment 3; Handout 4, 20 items of proverbs for Experiment 4.

For each of the experiments, three kinds of the test were conducted: the pretest was designed for measurement of lexical competence on the part of L2 or FL learners before the experimental treatment, the immediate posttest for short-term recall, and the one-week delayed posttest for middle-term retention. After four week's teaching and learning, the final comprehensive test was conducted to test the comprehensive effect. Six months later, the follow-up with TAPs included was carried out to confirm the long-term retention.

The procedures for each of the experiments were the same, which consisted of the pretest (the test before learning) of 15 minutes, the immediate posttest (the test immediately after learning) of 15 minutes, and the one-week delayed posttest (the test after learning for one week) of 15 minutes. The teaching materials used in each of the experiments were the same for both the CC and the EC. The only difference between the CC and the EC was that the two different approaches were adopted in the process of teaching and learning.

For the CC, the targeted materials were listed at random, and no information was provided regarding the metaphorical nature or schematic feature. The teaching treatment for this group was mainly by GT-method, the traditional grammar and translation teaching approach. The conceptual metaphor theory and the image schema theory were not involved.

For the EC, the cognitive linguistics-inspired approach was adopted, in which the non-arbitrary nature of the target materi- als was explored, including the schematic features and conceptual mapping models between the underlying CMs and the IMs and the target materials grouped around their underlying schematic features and CMs on the handouts and the blackboard. Illustrations representing the major schematic feature and the major mappings of CM (e.g. from concrete domain to abstract domain,) were provided with the expressions targeted.

As an extra task, for the EG, the students were asked to identify common themes or conceptual metaphors (CMs) behind the linguistic expressions listed. The $\mathrm{CM}$ was provided with examples such as TIME is MONEY and THEORIES are BUILDINGS or ARGUMENT is A WAR.

The interaction between students and their instructor included guided discussion, questions and answers, reading, writing, defining, paraphrasing as well as translation in the process of experiments for information on various aspects of raising $\mathrm{CM}$ and IM awareness.

After classroom treatment, the immediate posttest and the delayed posttest were conducted, with Minitab one-way ANOVA to check if there is any significant difference statistically found between the two classes.

\section{Participants}

The 57 student participants all came from the four natural parallel classes of third year English majors from the Translation Department, School of English Studies, Tianjin Foreign Studies University. They were aged at $19-22$ and all of them had passed the National English Major Level 4 Test (EMT-4), and their proficiency in English can be leveled as advanced EFL learners.

To make the comparative experiments valid, the participants were divided into two experimental teaching classes: Class 1 as an Experimental Class (EC), consisting of 28 students, to be taught via the proposed CL-inspired approach; Class 2 as Control Class (CC), consisting of 29 students, to be taught via GT-method.

According to the questionnaire and informal interview before the classroom experiments, the participants from the two classes were eager to learn and very willing to be involved in the experiment. All of them registered in the course offered for juniors by the current researcher. There would be no problem for the present researcher to be in touch with them for the feedback related to the experiments.

\section{Materials}

The materials used for experimental treatment were mainly chosen from Conceptual Metaphor Home Page http://cogsci. berkeley.edu/lakoff and from the book by Milada Broukal (1995) Idioms for Everyday Use by Houghton Mifflin Company; the American Heritage Dictionary of Idioms for Students of English (2002); the monographs by Lakoff (1980) Metaphors We Live by. Chicago: The University of Chicago Press; Lakoff and Johnson (1987) Women, Fire, and Dangerous Things: What Categories Reveal about the Mind. Chicago: University of Chicago Press; Langaker R. W. (1987) Foundations of Cognitive Grammar: Theoretical Prerequisites (Volume I) Stanford: Stanford University Press; and the idioms and proverbs used in Gibbs, R.'s (1992) What Do Idioms Really Mean? Journal of Memory and Language, 31, 485-506; and Li, Fuyin's (2004) Applied Cognitive Linguistics, Beijing: China Wenshi Publishing, altogether including 20 lexical metaphors; 20 verb-particle structures; 20 English idioms, and 20 English proverbs. 


\section{Collecting and Analyzing Data}

\section{Date Collection}

The data used in this study were mainly collected from the two tests of the linguistic proficiency; the three tests of the four classroom experiments; and one final comprehension test to measure the comprehensive achievements of the participants treated with different methods and approaches, which was conducted at the end of the four experiments and one follow-up survey with TAPs included, carried out six months after the experiments.

The proficiency tests were required to evaluate the participants' proficiency in English before the experiments, with an aim to certify that the two classes are at the same level of language fluency, the one of which is the EMT-4, conducted on December 19, 2008, which was administered by the Chinese National Educational Committee; and the other one is the DEICT, conducted on May 19, 2009, which was designed and carried out before the classroom experiments by the current researcher; the three tests for each of the four classroom experiments, i.e. pretest, immediate posttests, delayed posttests, which were designed to measure the effect of the method and the approach adopted in the experiment, implemented during the classroom experiments. To be more specific, the pretests of the four classroom experiments were designed to test the students' vocabulary competence and identify whether or not they had learned the target materials before the classroom teaching, the contents for these tests cover the materials to be targeted during the classroom teaching, including 20 items of lexical metaphors for Experiment 1; 20 items of verbal-particle (VP) constructions for Experiment 2; 20 items of idioms for Experiment 3; 20 items of proverbs for Experiment 4 ; the immediate posttests were designed to measure the effect of the method and the approach adopted in the experiment in terms of the short-term memory, and the contents for these tests cover the same materials used for the pretests described above; the delayed posttests were designed to measure the effect of the method and the approach adopted in the experiment in terms of the mid-term memory, and the test material involved what they had learned within the 60 minutes of the experimental teaching; The final comprehensive test was designed to measure the comprehensive achievements on the part of the participants in terms of the middle-term retention, conducted at the end of the four classroom experiments, the contents of which cover all the materials targeted in the four classroom teaching with respect to comprehension and retention, metaphorical competence, pragmatic or socio-linguistic competence and positive affect. The results of the final test could be essential evidence for either supporting or not supporting the primary hypothesis formulated in the beginning of the present study; the follow-up survey with think-aloud protocols included is designed to confirm the effectiveness of the CL-inspired approach on L2 or FL acquisition in terms of the long-term retention. The follow-up survey, conducted six months after the classroom experiments, covers the similar materials used for the final comprehension test, i.e. all the materials targeted in the four classroom experiments. The questionnaires were also used in the form of think-aloud protocol to get the comprehensive feedback of the experimental treatment.

All the data collected from the tests above, including: (a) the two proficiency tests; (b) the four pretests, the four immediate-posttests and the four delayed-posttests; (c) the one final comprehensive test; (d) the one follow-up test, with TAPs included, were analyzed with Minitab 15 One-way ANOVA within a prescriptive statistical framework. The detailed analysis was presented in 5.2 .

\section{Data Analysis}

Minitab One-Way ANOVA versus Class: Proficiency Test

The outcome of Minitab One-way ANOVA versus Class for Proficiency test shows that the EMT- 4 versus Class yields $\mathrm{p}(=0.804)>.05$, with a mean score of $70.071 \%$ for the EC and $69.621 \%$ for the CC, and the DEICT versus Class yields $\mathrm{p}$ $(=0.600)$, with a mean score of $74.679 \%$ for the EC and 75.793\% for the CC (See Table 1).

\section{Minitab One-Way ANOVA versus Class: Four Classroom Experiments}

\section{One-Way ANOVA for Exp 1}

The outcome of Minitab One-way ANOVA versus Class for experiment 1 (Exp 1) of the four classroom experiments yields $\mathrm{p}(=0.389)>.05$, with a mean score of $15.179 \%$ for the EC and $15.724 \%$ for the CC in the pretest; the immediate posttest yields at $\mathrm{p}(=0.005)<.05$, with a mean score of $54.26 \%$ for the EC and $73.79 \%$ for the CC; the delayed posttest yields at $\mathrm{p}(=0.000)$ $<.05$, with a mean score of $64.46 \%$ for the EC and $42.76 \%$ for the CC (See Table 2).

\section{One-Way ANOVA versus Class for Exp 2}

The outcome of Minitab One-way ANOVA versus Class for Exp 2 yields $p(=0.292)>.05$, with a mean score of $18.089 \%$ for the EC and $17.336 \%$ for the CC in the pretest; the immediate posttest yields $p(=0.196)>.05$, with a mean score of 74.64 for the EC and 69.66 for the CC; the delayed posttest yields $\mathrm{p}(=$ $0.038)<.05$, with a mean score of $65.18 \%$ for the EC and $58.45 \%$ for the CC (See Table 3).

\section{One-Way ANOVA versus Class for Exp 3}

The outcome of Minitab One-way ANOVA versus Class for Exp 3 yields $p(=0.723)>.0 .5$, with a mean score of $18.393 \%$ for the EC and $18.147 \%$ for the CC in the pretest; the immediate posttest yields $\mathrm{p}(=0.022)<.05$, with a mean score of $85.85 \%$ for the EC and $79.48 \%$ for the CC; the delayed posttest yields $\mathrm{p}(=0.017)<.05$, with a mean score of $82.41 \%$ for the EC and $75.80 \%$ for the CC ( See Table 4 ).

One-Way ANOVA versus Class for $\operatorname{Exp} 4$

The outcome of Minitab One-way ANOVA versus Class for Exp 4 yields $p(=0.341)>.05$, with a mean score of $14.948 \%$ for the EC and $14.259 \%$ for the CC in the pretest; the immediate

Table 1.

Means and $P$ value for the proficiency test.

\begin{tabular}{ccccc}
\hline Class & Number & EMT-4 (m) & DEICT (m) & EMT-4 (p value) \\
\hline EC & 28 & 70.071 & 74.679 & 0.804 \\
CC & 29 & 69.62 & 75.79 & 0.600 \\
\hline
\end{tabular}


Table 2.

Means and $p$ value of Exp 1

\begin{tabular}{cccc}
\hline & EC $(\mathrm{m})$ & CC $(\mathrm{m})$ & p value \\
\hline Pretest & 15.179 & 15.724 & 0.389 \\
Imm.-posttest & 54.26 & 73.79 & 0.005 \\
Delayed posttest & 64.46 & 42.76 & 0.000 \\
\hline
\end{tabular}

Table 3.

Means and $p$ value of Exp 2.

\begin{tabular}{cccc}
\hline & EC $(\mathrm{m})$ & CC $(\mathrm{m})$ & p value \\
\hline Pretest & 18.089 & 17.336 & 0.292 \\
Imm.-posttest & 74.64 & 69.66 & 0.196 \\
2-week posttest & 65.18 & 58.45 & 0.038 \\
\hline
\end{tabular}

Table 4.

Means and $p$ value of Exp 3.

\begin{tabular}{cccc}
\hline & EC $(\mathrm{m})$ & CC $(\mathrm{m})$ & p value \\
\hline Pretest & 18.393 & 18.147 & 0.723 \\
Imm.-posttest & 85.85 & 79.48 & 0.022 \\
3-week posttest & 82.41 & 75.80 & 0.017 \\
\hline
\end{tabular}

posttest yields $\mathrm{p}(=0.000)<.05$, with a mean score of $89.214 \%$ for the EC and $82.310 \%$ for the CC; and the delayed posttest yields $\mathrm{p}(=0.000)<.05$, with a mean score of $64.46 \%$ for the EC and $42.76 \%$ for the CC (See Table 5).

One-Way ANOVA versus Class: The Final Comprehensive Test

The outcome of Minitab One-way ANOVA versus Class for the final comprehensive test shows that Part 1 (lexical metaphor competence) yields $\mathrm{p}(=0.002)<.05$, with a mean score of $8.535 \%$ for the EC and $7.827 \%$ for the CC ; Part 2 (grammatical competence) yields $\mathrm{p}(=0.021)<.05$, with a mean score of $5.304 \%$ for the EC, and $4.138 \%$ for the CC; Part 3(semantic competence) yields $\mathrm{p}(=0.014)<.05$, with a mean score of $27.679 \%$ for the EC, and $25.966 \%$ for the CC; Part 4 (pragmatic competence) yields $\mathrm{p}(=0.015)<.05$, with a mean score of $22.464 \%$ for the EC, and $20.103 \%$ for the CC; Part 5 (translation competence) yields $\mathrm{p}(=0.031)<.05$, with a mean score of $16.946 \%$ for the EC, and $15.914 \%$ for the CC. Totally, significant difference has been found at p value $(=0.016)<0.5$, with the mean score of $80.928 \%$ for the EG, and $73.948 \%$ for the CG (See Table 6).

One-Way ANOVA versus Class: The Follow-up Survey

The outcome of Minitab One-way ANOVA versus Class for the follow-up survey shows that Part 1 (lexical metaphor competence) yields $\mathrm{p}(=0.000)<.05$, with a mean score of $8.39 \%$ for the EC and $4.13 \%$ for the CC; Part 2 (grammatical competence) yields $\mathrm{p}(=0.047)<.05$, with a mean score of $9.03 \%$ for the EC, and $8.65 \%$ for the CC; Part 3 (semantic competence) yields $\mathrm{p}$ $(=0.000)<.05$, with a mean score of $23.50 \%$ for the EC, and $18.03 \%$ for the CC; Part 4 (pragmatic competence) yields p $(=0.000)<.05$, with a mean score of $24.85 \%$ for the EC, and $15.31 \%$ for the CC; Part 5 (translation competence) yields $p$ $(=0.011)<.05$, with a mean score of $16.37 \%$ for the EC, and
$12.60 \%$ for the CC. Totally, significant difference has been found at $p$ value $(=0.011)<0.5$, with the mean score of $82.160 \%$ for the EG, and $58.737 \%$ for the CG (See Table 7).

\section{Results and Discussion}

\section{Proficiency Test}

Based on the Minitab one-way ANOVA versus Class for language proficiency tests, EMT-4 yields $\mathrm{p}(=0.804)>.05$, with the mean score of 70.071 percent for EC and 69.621 for CC, and DICT versus Class yields $p(=0.600)>.05$, with the mean score of 74.679 percent for EC and 75.793 percent for CC.

The results suggest that the participants from the two classes are at the same level in terms of their EFL fluency, thus, they are comparable in the present study.

It follows that if there is any difference between the two classes after the experimental teaching, it should be attributed to the effects of the methods or approaches adopted in teaching and learning.

\section{Three Tests of the Four Classroom Experiments}

\section{Experiment 1 (EXP1)}

Based on the Minitab one-way ANOVA versus Class for

Table 5.

Means and $p$ value of Exp 4

\begin{tabular}{cccc}
\hline & EC $(\mathrm{m})$ & CC $(\mathrm{m})$ & $\mathrm{p}$ value \\
\hline Pretest & 14.948 & 14.259 & 0.341 \\
Imm.-posttest & 89.214 & 82.310 & 0.000 \\
Delayed posttest & 64.46 & 42.76 & 0.000
\end{tabular}

Table 6 .

Means and $p$ value of the final test.

\begin{tabular}{cccc}
\hline & EC $(\mathrm{m})$ & CC $(\mathrm{m})$ & p value \\
\hline Part-1 & 8.535 & 7.827 & 0.002 \\
Part-2 & 5.304 & 4.138 & 0.021 \\
Part-3 & 27.679 & 25.966 & 0.014 \\
Part-4 & 22.464 & 20.103 & 0.015 \\
Part-5 & 16.946 & 15.914 & 0.031 \\
Total & 80.92 & 73.94 & 0.016 \\
\hline
\end{tabular}

Table 7.

Means and $p$ value of the follow-up survey.

\begin{tabular}{cccc}
\hline & EC $(\mathrm{m})$ & CC $(\mathrm{m})$ & p value \\
\hline Part-1 & 8.39 & 4.13 & 0.000 \\
Part-2 & 9.03 & 8.65 & 0.047 \\
Part-3 & 23.50 & 18.03 & 0.000 \\
Part-4 & 24.85 & 15.31 & 0.000 \\
Part-5 & 16.37 & 12.60 & 0.011 \\
Total & 82.16 & 58.73 & 0.011 \\
\hline
\end{tabular}


EXP1, the results from the pretest show no significant difference statistically found between the two classes, suggesting that the participants were at the same level in terms of their lexical metaphor competence, thus if any difference was found after teaching, the methods or approaches adopted in the experiment should take effect.

Surprisingly, the mean score of the EC from the immediate posttest is lower than that of the CG by 19.53 percent, unwillingly suggesting that there is no superiority at all for the CL-inspired approach adopted in term of short-term recall.

Interestingly, when it comes to the results of the delayed posttest, the mean score of the EC is higher than that of the CC by 21.7 percent, with the $\mathrm{p}$ value showing significant difference found between the two classes. That is, the EC are forgetting less than the CC, willingly suggesting that CL-inspired approach works much better than the traditional GT-method in terms of middle term retention.

An informal oral interview after class found out that undesirable short-term recall of the EC might be caused by lack of warming-up activities and limited time for the new approach adopted in the experiment. Considering that both EFL learners and the instructor were trained with the traditional teaching method before the experiments, it might take a while for them to get used to the newly proposed approach. In this case, the CL-inspired approach failed to work in the way as expected, particularly for the short-term recall of the target materials.

Regarding the fact that the mean score of the EC is lower than that of the CG by 19.53 percent from the immediate posttest, this study holds that given extra-variables, such as style of teaching, time spent for the classroom input also play roles in the process of learning based on the information from the informal interview of the participants.

Putting all the facts into consideration, conclusion can be made that in terms of lexical metaphoric competence, the superiority of the CL-inspired approach is still available since significant difference has been found statistically between the two classes with respect to the middle term recall. In another word, the CL-inspired approach works better than the traditional GT-method unless the treatment is affected with extra-variables, such as style of teaching, time spent for the classroom input.

\section{Experiment 2 (EXP2)}

Based on the Minitab one-way ANOVA versus Class for EXP2, the results from the three tests show that there is no significant difference found between the two classes from the pretest, suggesting that the participants were at the same level in terms of their VP construction competence, thus if any difference found after teaching, the methods or approaches adopted in the experiment should take effect.

Unwillingly, no significant difference statistically found between the two groups from immediate-posttest, but, with the mean score of the EC higher than that of the CC by 4.98 percent, suggesting that the CL-inspired approach works a little better despite the fact that there is no significant difference found statistically;

It is more encouraging that statistically significant difference found between the two classes from the delayed posttest, with the mean score of the EC higher than that of the CC by 6.73 percent, suggesting that the proposed CL-inspired approach shows its superiority over the traditional GT-method in terms of middle-term retention.

Referring both the p value and the mean score for each of the two classes, it has been found that the difference between the two classes is increasing in favor of the EC since with the $\mathrm{p}(=0.196)>.05$ in the immediate posttest, the participants from the EC outperformed the CC by 0.75 percent and with the $\mathrm{p}(=0.038)<.05$ in the delayed posttest, the participants from the EC outperformed the CC by 6.73 percent.

It follows that the CL-inspired approach works in the potential manner facilitating learners in the process of leaning English as L2 or FL in terms of the VP constructions with respect to comprehension and retention of memory.

\section{Experiment 3 (EXP3)}

Based on the Minitab one-way ANOVA versus Class for EXP3, the results from the three tests show that there is no significant difference found between the two classes from the pretest, suggesting that the participants were at the same level in terms of their idiomatic competence, thus if any difference was found after teaching, the methods or approaches adopted in the experiment should take effect.

Encouragingly, significant difference has been statistically found between the two classes from both the immediate posttest and the delayed posttest, with $\mathrm{p}(=0.022)>.05$ in the immediate posttest and with the $\mathrm{p}(=0.017)<.05$ in the delayed posttest, a mean score of EC higher than that of CC by 6.37 percent in the immediate posttest and by 6.61 percent in the delayed posttest, suggesting that the EC are forgetting less than the CC.

It follows that the CL-inspired approach shows its superiority over the GT-method in a dominant way not only in terms of middle-term retention, but also short-term recall as well in the process of learning advanced usage of vocabulary in terms of idiomatic expressions with respect to comprehension and retention of memory.

\section{Experiment 4 (EXP4)}

Based on the Minitab one-way ANOVA versus Class for EXP4, the results from the three tests show that there was no significant difference found between the two classes from the pretest, suggesting that the participants are at the same level in terms of their proverbial expression competence; thus if any difference was found after teaching, the methods or approaches adopted in the experiment should take effect.

Desirably, significant difference has been statistically found between the two classes from both the immediate posttest and the delayed posttest, with $\mathrm{p}(=0.000)>.05$ in the immediate posttest and with the $\mathrm{p}(=0.000)<.05$ in the delayed posttest, a mean score of EC higher than that of CC by 6.90 percent in the immediate posttest and by 21.7 percent in the delayed posttest, suggesting that the EC are forgetting less than the CC.

It follows that the CL-inspired approach shows its superiority over the GT-method in a very dominant way not only in terms of middle-term retention, but also short-term recall as well in the process of learning advanced usage of vocabulary in terms of proverbial expressions with respect to comprehension and retention of memory.

\section{Final Comprehensive Test}

Based on the Minitab one-way ANOVA versus Class for the final comprehensive test, the results from the final comprehensive test can be presented as below:

Part 1: significant difference has been found between the two groups in terms of grammatical competence, with $\mathrm{p}$ value $(=0.002)<.05$, a mean of 8.535 percent for the EC and of 7.827 percent for the CC; Part 2: significant difference has been found between the two groups in terms of metaphorical competence, with p value $(=0.021)<.05$, a mean of 5.304 percent for the EC; of 4.138 percent for the CC; Part 3: significant difference has been found between the two groups in terms of semantic com- 
petence, with $\mathrm{p}$ value $(=0.014)<.05$, a mean of 27.679 percent for the EC; of 25.966 for the CC; Part 4: significant difference has been found between the two groups in terms of pragmatic competence, with $\mathrm{p}$ value $(=0.015)<.05$, a mean of 22.464 percent for the EC; of 20.163 for the CC; Part 5: significant difference has been found between the two groups in terms of translation competence, with p value $(=0.031)<.05$, a mean of 16.946 percent for the EC; of 15.914 for the CC; Totally, the participants treated with the CL-inspired approach outperformed those taught with GT-method, with p value $(=0.016)$, a mean score of EC higher than that of CC by 6.332 percent.

Evidently, the primary hypothesis of present study has been verified with the results from the final comprehensive test, which is conducted at the end of the classroom experiments.

\section{Follow-up Survey and Think-Aloud Protocols}

As described in 5.1, the follow-up survey with think-aloud protocols included are designed to confirm the effectiveness of the CL-inspired approach on L2 or FL acquisition in terms of the long-term retention. The results from the test and thinkaloud protocols can be presented as below:

Part 1: significant difference has been found between the two classes in terms of lexical competence, with p value $(=0.000)$ $<.05$, a mean of 8.39 percent for the EC; of 4.13 percent for the CC; Part 2: significant difference has been found between the two classes in terms of grammatical competence, with $\mathrm{p}$ value $(=0.047)<.05$, a mean of 9.03 percent for the EC; of 8.65 percent for the CC; Part 3: significant difference has been found between the two $g$ classes in terms of semantic comprehension competence, with p value $(=0.000)<.05$, a mean of 23.50 percent for the EC; of 18.03 percent for the CC; Part 4: significant difference has been found between the two classes in terms of pragmatic competence, with p value $(=0.000)<.05$, a mean of 24.85 percent for the EC; of 15.31 percent for the CC; Part 5: significant difference has been found between the two classes in terms of translation competence, with p value $(=0.011)<.05$, a mean of 16.37 percent for the EC of 12.60 percent for the CC; Totally, significant difference statically found totally, with $\mathrm{p}$ $(=0.011)<.05$, a mean of 82.16 percent for the EC; 58.73 percent for the CC in terms of long-term retention, suggesting that the participants treated with the CL-inspired approach outperformed those taught with GT-method with p value $(=0.011)$, a mean score of EC higher than that of CC by 23.43 percent.

It follows that the effectiveness of the CL-inspired approach in the process of EFL acquisition has been confirmed with respect to the short-term recall, middle-term retention, and the long-term retention of memory.

To sum up, based on the Minitab one-way ANOVA versus Class, the EC, treated with the CL-inspired approach, outperformed the CC, taught with the GT-method, by 21.7 percent in EXP1; 6.73 percent in EXP2; 6.61 percent in EXP3; 21.7 percent in EXP4, 6.98 percent in the final, and 23.43 percent in the follow-up based on the mean score of classroom experiment delayed posttests and the total results of the final comprehensive test as well as the follow-up test. Evidently, the primary hypothesis formulated in the beginning of the present study has been experimentally and statistically verified with the empirical evidence from the four experiments, the final, and the follow-up, i.e. the CL-inspired pedagogy facilitates L2 or FL acquisition in terms of advanced usage of vocabulary such as lexical metaphors, verb-particle constructions, idioms and proverbs with respect to comprehension and retention of memory.

If we explore the reason why, the following factors can be put into consideration:
In the first place, the CL-inspired approach is mainly based on the conceptual metaphor theory (CMT) and the image schema theory (IMT), supported with DCT and the trace theory of memory, thus, the conceptual metaphor (CM) and the image schema (IM) play an essential role in this approach.

Essentially, recognition of CMs and image schematic features benefits learners in understanding target materials in general and recognizing persuasive rhetoric in particular. The ability to identify the source domains behind the linguistic forms also helps students recognize the links between the form and the meaning. In other words, metaphor and image schematic analysis uncovers the underlying relationship between the original or literary meanings and metaphorical or figurative meanings of the target materials, thus, enhancing their comprehension, retention and pragmatic or socio-cultural competence.

In CL theory, the CM is conceived of as a belief structure, which is defined as understanding one conceptual domain in terms of another conceptual domain. For instance, we talk, understand and construe the concept TIME in terms of MONEY; IDEA in terms of FOOD; ECONOMY in terms of BUILDING; RELATIONSHIP in terms of JOURNEY; and many others. In CMT, there is a cross-domain mapping from the concrete source domain (e.g. MONEY) to the abstract target domains (e.g. TIME), formulated as a CM: TIME IS MONEY.

Accordingly, a cluster of linguistic expressions may be produced under this $\mathrm{CM}$, such as waste time, use up time, run out of time, save time, spend time, gave time, budget for time, invest time, no time left, devote time, etc. If we analyze these expressions in a reversal way, all of them are organized under and relatable to a single conceptual metaphor: TIME IS MONEY (Gao, 2010: p. 112).

If language learners can be made aware of the metaphoric nature of many lexical expressions and verbal particle constructions by referring to their literal (or original) senses, take dead metaphors for example, this kind of metaphor can be resuscitated by associating the idiomatic expressions with a more vivid or concrete scene. The results from Experiment 1 of present study have confirmed this beneficial affect.

Secondly, the effectiveness of the CL-inspired approach on EFL acquisition gets empirical support from the DCT proposed by Pavio (1965, 1972, 1977), and the Trace theory of memory.

DCT attempts to explain cognition, comprehension and memory in terms of two systems: one is the verbal system specialized for dealing with language, and the other is the nonverbal system (called the imagery system) for representing and processing world knowledge about objects and events. According to this theory, information is stored in two codes, the verbal information in the verbal system, and the imagery information in the imagery system (Paivio, 1969, 1981, 1986, 1991; Gee et al., 1999). The central implication of DCT is the concreteness effect on memory: concrete words are easier to recall due to the way they are coded.

This study holds that DCT can provide supports for the hypothesis that the proposed CL-inspired pedagogy facilitates L2 or FL acquisition in that it assumes that information stored mentally in two codes should be better comprehended and remembered than information stored only in one code, i.e. the dual coding evidently enhances the trace of memory, resultantly, the students treated with the CL-approach forget less than those treated with the GT-method.

Thirdly, identifying the metaphoric and schematic features underlying the lexical metaphors, verb-particle phrases, idioms and proverbs can facilitate comprehension and enhance retention, since once the underlying metaphor and image schemas 
(e.g., the CONTAINER-schema) are activated, they will start to function as metaphors and image schemas and all superior functions of metaphors and image schemas in memory and learning will start to work. What is most important to this study is that IMs represent the essential glue that binds embodied experience, thought, and language, thus, they have a potential value in facilitating comprehension and long term retention of memory.

According to Linder's (1983) explanation that both the verb and the particle not only contributes semantically to the verb-particle construction, but also provide reasons for some of the syntactic and semantic limitations and constraints found in the usage of patterns of the complete constructions. As illustrated above, the spatial words contained expressions are arranged surrounding their basic conceptions with schematic pictures. The level of specificity at which the semantics of phrasal verbs is motivated might facilitate dual coding in the learners' mind with precise, rich images (Boers, 2004).

The following examples are quoted from Johnson (1987: p. 34), which further illustrated how a single image schema, as a recurring organizing structure, can help us understand and structure different kinds of experiences and reason about them. They are instances of metaphorical projections of the CONTAINER schema in our understanding of abstract states. The sentences in $(1$ - 3) show the case in point:

1) Tell me a story again, but leave out the minor details. (STORY EVENT AS CONTAINER)

2) I give up; I'm getting out of the race. (RACE EVENT AS CONTAINER)

3) Whenever I'm in trouble, she always bails me out. (STATE AS CONTAINER)

As noted, the abstract states are interpreted as spatially bounded entities or locations. The power of image schemas lies in the fact that we can metaphorically extend them from the physical to the nonphysical so as to structure and order our experience in abstract domains.

From the result of this experiment, we can draw the conclusion that a combination with DUAL CODING THEORY and IMAGE SCHEMA can facilitate L2 learners' vocabulary expansion effectively.

Moreover, identifying certain schematic features of VP construction can facilitate dual coding because of our experience with objects INSIDE and OUTSIDE. This boundedness of entity allows for CONTAINRER-schema transformation to give rise to a rotated sense, thus enhancing comprehension and retention of memory.

Take the sentence "John went out of the room" for example. This typical OUT movement based CONTAINER-schema can represent an enormous number of orientation possibilities. e.g., (1-17):

1) Mary got out of the car; 2) Spot jumped out of his pen; 3) He squeezed out some toothpaste; 4) Tear out that cartoon and save it; 5) Get out of bed; 6) John went out of the room; 7) Pump out the air; 8) Let out your anger; 9) Pick out the best theory; 10) Drown out the music; 11) Barry weaselled out of the contract; 12) Pour out the beans; 13) Roll out the red carpet; 14) Send out the troops; 15) Hand out the information; 16) Write out your ideas; 17) The train started out for Chicago.

Fourthly, making the learners aware of the concrete domain in metaphorical mapping and recognize the schematic feature of the target materials enhance their comprehension and retention according to the DCT since CM and IM can help the L2 or FL learners to form image easily.

For instance, the abstract concepts like being out of rage can be illustrated by drawing a picture to show the students the scene of an uncontrollable fire, and the concepts like running out of steam can be illustrated by drawing a picture of box to show the students the feature of Container-image schema. The advantage of this technique is that it evokes imagery of concreteness to facilitate vocabulary learning (e.g., Sokmen, 1997).

This effect, in turn, can be explained by reference to the dual coding hypothesis (e.g., Clark \& Paivio, 1991), which maintains that concrete items are actually stored twice, verbally as well as non-verbally (i.e., as an image). That is, if teachers systematically draw the attention of language learners to the source domains of linguistic metaphors and of vocabulary involving metaphor, then the learners' depth of knowledge for that language, and their ability to retain it can improve systematically (e.g, Boers, 2000, quoted from Littlemore, 2006).

Last but not least, an awareness that language need not be learned entirely via a long and daunting road of blind memorization has been proven to be an encouraging thought to learners who recognize that large amount of the target language actually "make sense" under the CL approach, i.e. through metaphorimage-schematic feature analysis. This encouragement has been paid off, considering the fact that the students treated with the CL-approach have been more involved in the experiments and even after the experiments in terms of class activities and informal interview and response to the follow up survey based on the observation of the present researcher. There is no doubt that such positive affect will be definitely beneficial to longer-term learning behavior.

\section{Conclusion and Implications}

The CL-inspired approach proposed in this study is mainly based on the insightfulness of the CL theories, i.e. conceptual metaphor theory (CMT) and the image schema theory (IMT), which is strongly supported with the dual coding theory (DCT) and the trace theory of memory. The major hypothesis of this approach is that through awakening students' metaphoric awareness and analyzing features of the image schematic structure, the language learners can benift in the process of learning English as a L2 or FL with respect to the advanced usage of vocabulary in terms of lexical metaphors, VP constructions, idioms and proverbs with respect to comprehension and retention of memory, thus enhancing their metaphorical comprehension, pragmatic or socio-linguistic competence, and positive affect. The superiority of this approach has been experimentally and statistically verified with the empirical evidence from a series of classroom teaching experiments carried out on the campus of Tianjin Foreign Studies University.

Theoretically, the proposed cognitive-inspired approach provides empirical evidence for the insightfulness of cognitive linguistics and significant implications practically for the L2 or FL teaching and research, thus bridging the gap between the cognitive linguistic researches and applied linguistic studies.

\section{References}

Achard, M., \& Niemeier, S. (Eds.) (2004). Cognitive linguistics, second language acquisition, and foreign language teaching. Berlin: Mouton de Gruyter.

Arnold, J. (1999). Affect language learning. Cambridge: Cambridge University Press.

Baddeley, A. (1990). Human memory. London: Lawrence Erlbaum Asso- 
ciates.

Beréndi, M., Csábi, S., \& Kövecses, Z. (2008). Using conceptual metaphors and metonymies in vocabulary teaching. In F. Boers and S. Lindstromberg (Eds.), Cognitive Linguistic Approaches to Teaching Vocabulary and Phraseology (pp. 65-100). New York: Mouton de Gruyter.

Boers, F., \& Lindstromberg, S. (Eds.) (2008). Cognitive linguistic approaches to teaching vocabulary and phraseology. New York: Mouton de Gruyter.

Boers, F., \& Lindstromberg, S. (2006). Cognitive linguistics application in second or foreign language instruction: Rationale, proposals, and evaluation. In G. Kristiansen, et al. (Eds.), Cognitive Linguistics: $\mathrm{Cu}$ Rrent Applications and Future Perspectives (pp. 305-358). New York: Mouton de Gruyter. doi:10.1515/9783110197761.4.305

Boers, F., \& Lindstomberg, S. (2005). Finding ways to make phraselearning feasible: The mnemonic effect of alliteration. System, 33, 225238. doi:10.1016/j.system.2004.12.007

Boers, F., \& Demecheleer, M. (2004). Cross-cultural variation as a variable in comprehending and remembering figurative idioms. European Journal of English Studies, 8, 375-388. doi:10.1080/1382557042000277449

Boers, F. (2000a). Enhancing metaphoric awareness in specialised reading. English for Specific Purposes, 19, 137-147. doi:10.1016/S0889-4906(98)00017-9

Boers, F. (2000b). Metaphor awareness and vocabulary retention. Applied Linguistic, 21, 553-571. doi:10.1093/applin/21.4.553

Boers, F. (1999). When a bodily source domain becomes prominent: The joy of counting metaphor in the socio-economic domain. In R. W. Gibbs and G. J. Steen (Eds.), Metaphor in Cognitive Linguistics (pp. 47-56). Amsterdam and Philadelphia: John Benjamins.

Boers, F., \& Demecheleer, M. (1998). Cognitive semantic approach to teaching preposition. English Language Teaching Journal, 52, 197204. doi:10.1093/elt/52.3.197

Boers, F. (1996). Spatial prepositions and metaphor: A cognitive semantic journey along the up-down and the front-back dimensions. Tubingen: Gunter Narr Verlag.

Bolinger, D. (1971). The phrasal verb in English. Cambridge, MA: Harvard University Press.

Broukal, M. (1995). Idioms for everyday use. IL: NTC Publishing Group.

Cameron, L., \& Low, G. (1999). Researching and applying metaphor. Cambridge: Cambridge University Press.

Clark, J. M. \& Paivio, A. (1991). Dual coding theory and education. Educational Psychology Review, 3, 149-170. doi:10.1007/BF01320076

Cohen, P. R. (2000). Learning concepts by interaction. Technical Report, Computer Science Department, University of Massachusetts at Amherst.

Condon, N. (2008). How cognitive linguistic motivations influence the learning of the phrasal verbs. In F. Boers and S. Lindstromberg (Eds.), Cognitive Linguistic Approaches to Teaching Vocabulary and Phraseology (pp. 133-158). New York: Mouton de Gruyter.

Croft, W (1998). Mental representations. Cognitive Linguistics, 9, 151174. doi:10.1515/cogl.1998.9.2.151

Cuyckens, H., Dirven, R., \& Taylor, J., B. (Eds.) (2003). Cognitive approaches to lexal semantics. New York: Mouton de Gruyte.

Ellis, N. C., \& Freeman, D. L. (2006). Language emergence: Implications for applied linguistics-introduction to the special issue. Applied Linguistics, 27, 558-589. doi:10.1093/applin/aml028

Fauconnier, G. (1985). Mental spaces: Aspects of meaning construction in natural language. Cambridge, MA: The MIT Press.

Fauconnier, G., \& Turner, M. (2002). The way we think: Conceptual blending and the minds hidden complexities. New York: Basic Books. A Member of the Perseus Books Group.

Fillmore, C. (1985). Frames and the semantics of understanding. Quaderni di Semantica, 6, 222-254.

Geeraerts, D. (2010). Theories of lexical semantics. New York: Oxford University Press.
Gibbs, R. (1992). What do idioms really mean? Journal of Memory and Language, 31, 485-506. doi:10.1016/0749-596X(92)90025-S

Gibbs, R. W. (2006). Embodiment and cognitive science. Cambridge: Cambridge University Press.

Hampe, B., \& Grady, J. (2005). From perception to meaning: Image schemas in cognitive linguistics. Berlin/New York: Mouton de Gruyter. doi:10.1515/9783110197532

Johnson, M. (1987). The body in the mind: The bodily basis of meaning, imagination, and reason. Chicago: University of Chicago Press.

Kövecses, Z. (2005). Metaphor in culture: Universality and variation. London: Cambridge University Press.

Kövecses, Z. (2006). Language, mind and culture: A practical introduction. Oxford: Oxford University Press.

Kövecses, Z., \& Szabó, P. (1996). Idioms: A view from cognitive semantics. Applied Linguistics, 17, 326-355.

doi:10.1093/applin/17.3.326

Lakoff, G., \& Johnson, M. (1980). Metaphors we live by. Chicago: The University of Chicago Press.

Lakoff, G. (1987). Women, fire, and dangerous things: What categories reveal about the mind. Chicago: University of Chicago Press.

Langacker, R. W. (1987). Foundations of cognitive grammar: Theoretical prerequisites (Volume I). Stanford: Stanford University Press.

Lazar, G. (1996). Using figurative language to expand students' vocabulary. ELT Journal, 50, 43-51. doi:10.1093/elt/50.1.43

Lazar, G. (2003). Meaning and metaphors: Activities to practise figurative language. Cambridge: Cambridge University Press.

Lindner, S. (1981). A lexico-semantic analysis of English verb-particle constructions with UP and OUT. Unpublished doctoral dissertation, San Diego, University of California.

Littlemore, J. (2001a). Metaphoric competence: A language learning strength of students with a holistic cognitive style? TESOL Quarterly, 35, 459-491. doi:10.2307/3588031

Littlemore, J. (2001b). An empirical study of the relationship between cognitive style and the use of communication strategy. Applied Linguistics, 22, 241-265. doi:10.1093/applin/22.2.241

Littlemore, J., \& Low, G. (2006). Figurative thinking and foreign language learning. Basingstoke, UK: Palgrave MacMillan. doi:10.1057/9780230627567

Littlemore, J., \& Low, G. (2006). Metaphoric competence, second language learning, and communicative. Language Ability Applied Linguistics, 27, 268-294.

Radden, G., \& Panther, K.-U. (2004). Studies in linguistic motivation. New York: Mouton de Gruyter.

Sadoski, M., \& Paivio, A. (2001). A dual coding theory of reading and writing. NJ: Lawrence Erlbaum Associates, Inc., Publishers.

Sophia, S. (2008). Conceptual metaphoric meaning clues in two idiom presentation methods. In F. Boers and S. Lindstromberg (Eds.), Cognitive Linguistic Approaches to Teaching Vocabulary and Phraseology (pp. 101-132). New York: Mouton de Gruyter.

Gao, Y. M. (2010). Applying CL to L2 acquisition. Tianjin: Nankai University Press.

Gao, Y. M. (2001). Learn how to learn: Comparative research on learning style preferences. Teaching English in China, 4, 51-57.

Gao, Y. M. (1996). T.R.I. model and college English reading. Foreign Language World, 3, 34-37.

Li, F. Y. (2004). Applied cognitive linguistics. Beijing: China Wenshi Publishing.

Liang, X. B. (2000). Enlightenments of the cognitive linguistics on English vocabulary teaching. Foreign Language Teaching and Research, 2, 35-39.

Liu, Z. G. (2010). Cognitive linguistic view of language and principles for FL teaching. Foreign Language Research, 1, 10-16.

Yu, N. (1998). The contemporary theory of metaphor: A perspective from Chinese. Philadelphia: John Benjamins.

Wang, Y. (2006). A Survey to cognitive linguistics. Shanghai: Shanghai Foreign Language Education Press. 